\title{
Edad al primer parto y productividad lechera del ganado bovino Holstein en la costa central del Perú
}

\author{
Age at first calving and dairy productivity of Holstein cattle in the \\ central coast of Peru \\ Emmanuel Sessarego D., ${ }^{13}$, Juan Chávez C. ${ }^{2}$, Alberto Barrón L. ${ }^{2}$, \\ Angel Vásquez R. ${ }^{1}$, Manuel López C. ${ }^{2}$
}

\section{Resumen}

El objetivo del estudio fue determinar el efecto de la edad al primer parto (EPP) sobre la producción estandarizada de leche (PEL) de la primera y segunda lactación e intervalo entre los primeros dos partos (IEP) en vacas Holstein de la cuenca lechera de Lima, Perú. Se analizaron registros de 4215 vacas provenientes de nueve establos, registrados en el Servicio de Productividad Lechera de la Universidad Nacional Agraria La Molina, entre enero de 2003 y diciembre de 2012. La información se analizó mediante estadística descriptiva y un modelo lineal mixto, utilizando el paquete SAS 9.4. La EPP promedio fue de $24.9 \pm 2.3$ meses. La EPP influyó en la PEL $(p=0.0008)$, pero no en el IEP $(p=0.3969)$. Asimismo, se encontraron diferencias significativas $(\mathrm{p}<0.01)$ entre las dos lactaciones, entre las cuatro estaciones del año y entre los 10 años evaluados, para las dos variables en estudio. En conclusión, se observaron mayores PEL y menores IEP a edades intermedias (22-30 meses), por lo que la EPP óptima estaría alrededor de los 22 meses para lograr maximizar el rendimiento productivo.

Palabras clave: edad al primer parto; producción de leche; intervalo entre partos; Holstein; costa central del Perú

\section{Abstract}

The aim of this study was to determine the effect of age at first calving (AFC) on the standardized production of milk (SPM) of the first and second lactation and interval between the first two calvings (CI) in Holstein cows of the Lima region, Peru. Records of

${ }^{1}$ E.P. de Ingeniería Zootécnica, Facultad de Ingeniería Agraria, Industrias Alimentarias y Ambiental, Universidad Nacional José Faustino Sánchez Carrión, Huacho, Perú

${ }^{2}$ Facultad de Zootecnia, Universidad Nacional Agraria La Molina, Lima, Perú

${ }^{3}$ E-mail: e.sessarego14@gmail.com

Recibido: 10 de enero de 2019

Aceptado para publicación: 18 de julio de 2019 
4215 cows from nine farms, registered in the Dairy Productivity Service of the National Agrarian University La Molina, between January 2003 and December 2012 were analysed. The information was analysed through descriptive statistics and by a mixed linear model, using SAS 9.4. The average AFC was $24.9 \pm 2.3$ months. The AFC influenced on the SPM $(p=0.0008)$, but not in the $C I(p=0.3969)$. Likewise, significant differences were found $(p<0.01)$ between the two lactations, between the four seasons of the year and between the 10 years evaluated for the two variables under study. In conclusion, higher SPM and lower CI were observed at intermediate ages (22-30 months), so the optimal AFC would be around 22 months to achieve maximum productive performance.

Key words: age at first calving; milk production; calving interval; Holstein; central coast of Peru

\section{INTRODUCCIÓN}

La crianza de animales de reemplazo es un proceso de larga duración y alto costo (Zanton y Heinrichs, 2005; Bouska et al., 2007), que en algunos casos representa hasta el $20 \%$ de los costos totales (Heinrichs, 1993). Este costo se puede reducir mediante la aceleración de la tasa de crecimiento y de la inseminación temprana, con lo cual se acorta la edad al primer parto (EPP) (Pietersma et al., 2006). En este sentido, los programas de crianza de vaquillas lecheras de reemplazo tienen como meta una EPP de 24 meses (Pirlo et al., 2000). Partos más allá de los 24 meses son de menor rentabilidad, dado el costo de manutención mensual de las vaquillonas (Bailey y Currin, 2009).

Bormann et al. (2002) señalan que vaquillonas que paren a una corta edad tienen una menor producción de leche durante su primera lactación; sin embargo, el rendimiento durante su vida productiva es significativamente mayor que el de vaquillas que tuvieron su primer parto a una edad más avanzada. De la misma manera, Meyer et al. (2004) indican que la reducción de la EPP puede incrementar la rentabilidad del establo. Por otro lado, Thompson et al. (1983) observaron que las distocias aumentaban significativamente cuando la EPP era menor a los 22 meses, mientras que Simerl et al.
(1992) no encontraron relación entre la EPP y el comportamiento reproductivo. Por último, se han reportado correlaciones moderadamente positivas entre la EPP y la producción de leche, grasa y proteína (Clark y Touchberry, 1962; Moore et al., 1991).

Con base a estos antecedentes, el objetivo del estudio fue evaluar la influencia de la EPP sobre la producción lechera e intervalo entre partos del ganado bovino Holstein de empresas ganaderas en la cuenca de Lima, durante el periodo 2003-2012.

\section{Materiales y Métodos}

\section{Lugar de ejecución}

El estudio se llevó a cabo en nueve establos lecheros ubicados en la región de Lima, Perú. El sistema de crianza de los establos es de tipo intensivo, con alimentación basada en forraje cortado y concentrado, detección visual de celos y empleo de inseminación artificial (IA) con semen congelado.

\section{Animales}

Como criterio de inclusión se consideraron solo los registros de vacas Holstein entre 20 y 36 meses al primer parto y que llegaron a su tercer parto; y como criterios 
Cuadro 1. Edad al primer parto, producción de leche e intervalo entre parto en vacas Holstein en establos lecheros de la costa central del Perú, durante el periodo 2003-2012 ( $\mathrm{n}=4215)$

\begin{tabular}{|c|c|c|c|c|}
\hline Variable & Media & D.E. & Min. & Max. \\
\hline Edad al primer parto (meses) & 24.9 & 2.3 & 20 & 36 \\
\hline Producción estandarizada de leche $(\mathrm{kg}), 1^{\text {ra }}$ lact. & $8,275.7$ & $1,471.1$ & 3,325 & 13,451 \\
\hline Producción estandarizada de leche $(\mathrm{kg}), 2^{\text {da }}$ lact. & $9,068.5$ & $1,747.0$ & 3,326 & 13,871 \\
\hline Intervalo entre partos (días), $1^{\text {ra }}$ lact. & 437.2 & 93.7 & 280 & 760 \\
\hline Intervalo entre partos (días), $2^{\text {da }}$ lact. & 447.4 & 94.4 & 289 & 760 \\
\hline
\end{tabular}

de exclusión las vacas con lactancias iniciadas por aborto o lacto-inducción, con lactancias terminadas debido a lesiones o enfermedades y con menos de 3000 o más de $14000 \mathrm{~kg}$ de leche en cada una de sus dos primeras lactancias.

\section{Variables e Información}

Se evaluó la edad al primer parto (EPP), la producción estandarizada de leche (PEL, 305 días y dos ordeños) y el intervalo entre partos (IEP, transformado a logaritmo para que cumpla con el supuesto de normalidad que exige el análisis de varianza); estas dos últimas variables, tanto para la primera como segunda lactación.

Se recolectaron 21075 datos, correspondientes a 4215 vacas, registrados por el Servicio de Productividad Lechera entre el 1 de enero de 2003 y el 31 de diciembre de 2012. Este servicio es conducido por el Programa de Investigación y Proyección Social en Mejoramiento Animal de la Universidad Nacional Agraria La Molina.

\section{Análisis Estadístico}

Para el análisis de los datos se utilizó el programa SAS v. 9.4. En primer lugar, se realizó un análisis descriptivo para las variables en estudio. Asimismo, para evaluar la influencia de la EPP sobre la PEL e IEP, se utilizó el siguiente modelo lineal mixto: $\mathrm{Y}_{\mathrm{ijklm}}=\mu+\mathrm{EPP}_{\mathrm{i}}$ + Parto $_{j}+$ Establo $_{\mathrm{k}}+$ Estación $_{1}+$ Año $_{\mathrm{m}}+$ $(\text { Parto*Establo })_{\mathrm{jk}}+(\text { Parto*Año })_{\mathrm{jm}}+$ $(\text { Establo*Año })_{\mathrm{km}}+($ Establo*Estación* Año $)_{\mathrm{klm}}+\varepsilon_{\mathrm{ijklm}}$, donde $\mathrm{Y}_{\mathrm{ijklm}}=$ Variable respuesta (PEL o Log. IEP), $\mu=$ Media general para las variables PEL o Log. IEP, EPP ${ }_{i}=$ Efecto de la $i$-ésima EPP (covariable), Parto $_{j}$ $=$ Efecto fijo del $j$-ésimo parto, Establo ${ }_{\mathrm{k}}=$ Efecto aleatorio del $k$-ésimo establo, Estación $=$ Efecto fijo de la l-ésima estación, Año $_{\mathrm{m}}=$ Efecto fijo del $m$-ésimo año, (Parto*Establo) ${ }_{j \mathrm{k}}$ $=$ Interacción entre el j-ésimo parto y el $k$ ésimo establo, (Parto*Año) $)_{\mathrm{jm}}=$ Interacción entre el j-ésimo parto y el $m$-ésimo año, $(\text { Establo*Año })_{\mathrm{km}}=$ Interacción entre el $k$ ésimo establo y el m-ésimo año, $(\text { Establo*Estación*Año) })_{\mathrm{klm}}=$ Interacción entre $k$-ésimo establo, l-ésima estación y $m$ ésimo año, y $\varepsilon_{\mathrm{ijklm}}=$ Error residual.

\section{Resultados y Discusión}

\section{Edad al Primer Parto}

La EPP en los establos evaluados estuvo bastante cercana al valor recomendado de 24 meses (Cuadro 1), lo cual estaría indicando que los establos logran preñar a la mayoría de sus vaquillas entre 14 y 15 meses. Este resultado es similar a lo reportado por Sessarego et al. (2014) para vacas del norte chico de Lima $(24.9 \pm 0.1$ meses $)$. Asi- 
mismo, es similar al reportado por la Asociación Israelí de Criadores de Ganado (ICBA, 2012) con 24 meses. Por otro lado, la EPP fue menor a lo obtenido por Pirlo et al. (2000), Evans et al. (2006), Scheneider et al. (2007), Wu et al. (2012) y Mohd Nor et al. (2013), quienes reportaron EPP de 28.1, 25.8, 28.4, 29.3 y 25.4 meses, para establos lecheros en Italia, Irlanda, Suecia, China y Holanda, respectivamente.

\section{Producción Estandarizada de Leche}

La PEL en los establos evaluados para la primera y segunda lactación fue de 8275 y $9068 \mathrm{~kg}$, respectivamente (Cuadro 1). La producción obtenida en la primera lactación fue comparativamente mayor que las reportadas por Pirlo et al. (2000), Muir et al. (2004), Rokouei et al. (2010) y Mohd Nor et al. (2013) de 7246, 7689, 7082 y $7518 \mathrm{~kg}$ en 305 días, para vacas primerizas Holstein en establos de Italia, Canadá, Irán y Holanda, respectivamente. Sin embargo, ligeramente menor a lo reportado en Pennsylvania, USA, de $8686 \mathrm{~kg}$ (Heinrichs y Jones, 2017) y mucho menor a la registrada en Israel $(12091 \mathrm{~kg}$; ICBA, 2012).

\section{Intervalo entre Partos}

Los IEP entre la primera y segunda lactación fueron de 437 y 447 días, respectivamente (Cuadro 1). Cabe resaltar que pese al costo adicional para el establo cuando las vacas no paren cada 365 días, en los EE. UU. se ha incrementado el IEP a través de los años (Hare et al., 2006), y la misma tendencia ha sido observada en Canadá (Murray, 2003), Holanda (KNRS, 2005) y Reino Unido (Wall et al., 2003).

\section{Efectos sobre la Producción de Leche}

La EPP influyó en la PEL ( $\mathrm{p}=0.0008$ ). En el Cuadro 2 se observa que vacas paridas entre 20 y 21 meses produjeron menos leche durante toda su campaña. Esta baja producción pudo estar relacionada con una alta tasa de crecimiento durante el periodo prepuberal, que ha demostrado afectar mayormente el desarrollo del tejido mamario (Sejrsen et al., 2000), aunque el factor más probable sería el bajo peso corporal de las vaquillonas muy jóvenes al primer parto (Hietanen y Ojala, 1995).

Por otro lado, vacas que parieron entre 22 y 36 meses de edad produjeron similares cantidades de leche (Cuadro 2). Tozer y Heinrichs (2001), a través de un estudio económico, mostraron que la reducción de la EPP, de 25 a 24 o a 21 meses reduce entre 4 y $18 \%$ el costo total de crianza de las vaquillas de reemplazo, mientras que el aumento de la EPP a 29 meses incrementa los costos en 14\%. La reducción de la EPP puede mejorar la rentabilidad de los establos lecheros, mediante el aumento de la vida productiva (Lin et al., 1988).

Se encontraron diferencias altamente significativas entre partos $(p<0.0001)$, entre estaciones $(p=0.0010)$ y entre años $(\mathrm{p}<0.0001)$ con relación a la producción estandarizada de leche.

\section{Efectos sobre el Intervalo entre Partos}

La EPP no influyó en el IEP ( $\mathrm{p}=0.3969$; Cuadro 3), resultado contrario al reportado por Evans et al. (2006), quienes observaron que vaquillonas Holstein que parían a los $25-$ 26 meses tenían una tendencia a disminuir los IEP posteriores en comparación con las que tenían mayor EPP. De igual manera, Ettema y Santos (2004) refieren que las tasas de concepción eran más bajas en la primera lactancia, tanto en las vaquillonas que parían temprano (<700 días, 23 meses) como las que parían tarde ( $>751$ días, 25 meses) en comparación con las que parían entre los 700 y 750 días.

Cooke et al. (2013) observaron que la fertilidad en la primera lactación era mejor en vaquillas que parían entre 23 y 25 meses y menor en las que parían después; sin embargo, no encontraron diferencias significativas de fertilidad entre las vacas de segunda lactación con diferentes EPP. Por el contra- 
Cuadro 2. Medias de cuadrados mínimos, error estándar e intervalo de confianza para la producción estandarizada de leche promedio de las dos primeras lactancias de vacas Holstein en la costa central de Lima, de acuerdo con la edad al primer parto

\begin{tabular}{cccccc}
\hline \multirow{2}{*}{$\begin{array}{c}\text { Edad al primer parto } \\
\text { (meses) }\end{array}$} & $\mathrm{n}$ & \multicolumn{4}{c}{ Producción estandarizada de leche $(\mathrm{kg})$} \\
\cline { 3 - 6 } & & $\mathrm{MCM}^{1}$ & $\mathrm{EE}^{1}$ & $\mathrm{IC}^{1}$ \\
\hline 20 & 17 & $7,528.7^{\mathrm{b}}$ & 254.3 & $7,030.2$ & $8,027.2$ \\
21 & 22 & $8,122.2^{\mathrm{ab}}$ & 223.8 & $7,683.5$ & $8,561.0$ \\
22 & 147 & $8,682.1^{\mathrm{a}}$ & 91.6 & $8,502.5$ & $8,861.7$ \\
23 & 929 & $8,526.1^{\mathrm{a}}$ & 46.4 & $8,435.2$ & $8,616.9$ \\
24 & 1,271 & $8,636.6^{\mathrm{a}}$ & 41.0 & $8,556.2$ & $8,717.1$ \\
25 & 701 & $8,653.4^{\mathrm{a}}$ & 47.8 & $8,559.8$ & $8,747.0$ \\
26 & 415 & $8,529.5^{\mathrm{a}}$ & 56.0 & $8,419.7$ & $8,639.4$ \\
27 & 247 & $8,604.4^{\mathrm{a}}$ & 69.6 & $8,468.0$ & $8,740.8$ \\
28 & 147 & $8,653.2^{\mathrm{a}}$ & 88.7 & $8,479.4$ & $8,827.1$ \\
29 & 105 & $8,591.5^{\mathrm{a}}$ & 103.1 & $8,389.4$ & $8,793.6$ \\
30 & 67 & $8,611.1^{\mathrm{a}}$ & 130.0 & $8,356.4$ & $8,865.8$ \\
31 & 41 & $8,222.4^{\mathrm{ab}}$ & 164.7 & $7,899.4$ & $8,545.3$ \\
32 & 30 & $8,396.5^{\mathrm{a}}$ & 192.8 & $8,018.6$ & $8,774.5$ \\
33 & 24 & $8,395.8^{\mathrm{a}}$ & 213.3 & $7,977.7$ & $8,814.0$ \\
34 & 13 & $8,910.1^{\mathrm{a}}$ & 290.5 & $8,340.8$ & $9,479.5$ \\
35 & 12 & $8,581.0^{\mathrm{a}}$ & 300.7 & $7,991.7$ & $9,170.4$ \\
36 & 27 & $8,501.3^{\mathrm{a}}$ & 203.8 & $8,101.8$ & $8,900.8$ \\
\hline
\end{tabular}

${ }^{1}$ MCM: media de cuadrado mínimo; EE: error estándar; IC: intervalo de confianza al $95 \%$

a,b Medias de cuadrados mínimos con letras diferentes dentro de columnas indican diferencias altamente significativas $(p<0.01)$

rio, Simerl et al. (1992) no hallaron efectos de la EPP sobre diversos parámetros reproductivos en vacas lecheras durante la primera lactación.

Estudios sobre curva de crecimiento en vacas Holstein hasta la tercera lactación muestran que los animales continúan creciendo durante este periodo, aunque la tasa disminuye luego de alcanzar los 450 días de edad (Coffey et al., 2006). Por tanto, vacas que paren muy jóvenes continúan creciendo en mayor medida después del parto, demandando un alto consumo de nutrientes, a expensas de la fertilidad. Por otro lado, vaquillonas que paren con una mayor EPP tienen mayor condición corporal, pero con consecuencias perjudiciales sobre la fertilidad (Wathes et al., 2008).
Se encontraron diferencias altamente significativas entre lactaciones $(\mathrm{p}=0.0004)$, entre estaciones del año $(\mathrm{p}<0.0001)$ y entre los 10 años $(\mathrm{p}<0.0001)$ con relación al intervalo entre partos.

\section{Conclusiones}

La edad al primer parto influyó en la producción estandarizada de leche $(\mathrm{p}=0.0008)$, observándose mejores producciones a edades intermedias ( 22 y 30 meses); sin embargo, la edad al primer parto no influyó en el intervalo entre partos $(\mathrm{p}=0.3969)$ en vacas Holstein en la costa central del Perú, durante el periodo 2003-2012. 
Cuadro 3. Medias de cuadrados mínimos, error estándar e intervalo de confianza para el intervalo entre partos promedio de las dos primeras lactancias de vacas Holstein en la costa central de Lima, de acuerdo con la edad al primer parto ${ }^{1}$

\begin{tabular}{cccccc}
\hline \multirow{2}{*}{$\begin{array}{c}\text { Edad al primer parto } \\
\text { (meses) }\end{array}$} & $\mathrm{n}$ & \multicolumn{4}{c}{ Intervalo entre partos (días) } \\
\cline { 3 - 6 } $\mathrm{EE}^{2}$ & $\mathrm{MCM}^{2}$ & \multicolumn{3}{c}{$\mathrm{IC}^{2}$} \\
\hline 20 & 17 & $449.0^{\mathrm{a}}$ & 15.9 & 417.8 & 480.2 \\
21 & 22 & $451.9^{\mathrm{a}}$ & 14.0 & 424.4 & 479.4 \\
22 & 147 & $444.7^{\mathrm{a}}$ & 5.7 & 433.4 & 455.9 \\
23 & 929 & $448.1^{\mathrm{a}}$ & 2.9 & 442.4 & 453.8 \\
24 & 1271 & $444.3^{\mathrm{a}}$ & 2.6 & 439.3 & 449.3 \\
25 & 701 & $448.2^{\mathrm{a}}$ & 3.0 & 442.4 & 454.1 \\
26 & 415 & $446.2^{\mathrm{a}}$ & 3.5 & 439.3 & 453.1 \\
27 & 247 & $449.9^{\mathrm{a}}$ & 4.4 & 441.3 & 458.4 \\
28 & 147 & $455.9^{\mathrm{a}}$ & 5.6 & 445.0 & 466.8 \\
29 & 105 & $450.2^{\mathrm{a}}$ & 6.5 & 437.6 & 462.9 \\
30 & 67 & $454.3^{\mathrm{a}}$ & 8.1 & 438.4 & 470.3 \\
31 & 41 & $450.0^{\mathrm{a}}$ & 10.3 & 429.7 & 470.2 \\
32 & 30 & $447.8^{\mathrm{a}}$ & 12.1 & 424.1 & 471.5 \\
33 & 24 & $477.1^{\mathrm{a}}$ & 13.4 & 451.0 & 503.3 \\
34 & 13 & $438.5^{\mathrm{a}}$ & 18.2 & 402.9 & 474.2 \\
35 & 12 & $418.2^{\mathrm{a}}$ & 18.8 & 381.3 & 455.1 \\
36 & 27 & $442.0^{\mathrm{a}}$ & 12.8 & 416.9 & 467.0 \\
\hline
\end{tabular}

${ }^{1}$ Para el análisis estadístico se transformaron los datos a su logaritmo, pero para su presentación en el cuadro se utilizaron los datos reales

${ }^{2}$ MCM: media de cuadrado mínimo; EE: error estándar; IC: intervalo de confianza al 95\%

a Medias de cuadrados mínimos con letras iguales dentro de columnas indican ausencia de diferencias altamente significativas $(p>0.01)$

\section{Agradecimientos}

Los autores expresan su gratitud a los establos lecheros en la región Lima que otorgan sus datos al Servicio de Productividad Lechera, conducido por el Programa de Investigación y Proyección Social en Mejoramiento Animal de la Universidad Nacional Agraria La Molina. Asimismo, agradecen al personal administrativo de dicho programa por su amabilidad y a todas aquellas personas que con su colaboración hicieron posible la presente investigación.

\section{Literatura Citada}

1. Bailey T, Currin J. 2009. Heifer inventory and the economics of replacement rearing. Virginia Cooperative Extension, Virginia State University. Virginia, USA. 4 p. [Internet]. Available in: https://www.pubs.-ext.vt.edu/content/dam/pubs_ext_-vt_edu/404/ 404-287/404-287_pdf.pdf

2. Bormann J, Wiggans GR, Gengler N, Druet T. 2002. Estimating effects of permanent environment, lactation stage, 
age and pregnancy on test-day yield. J Dairy Sci 85: 263-284. doi: 10.3168/ jds.S0022-0302(02)74076-9

3. Bouška J, Štípková M, Krejèová M, Bartoò L. 2007. The effect of growth and development intensity in replacement heifers on economically important traits of Holstein cattle in Czech Republic. Czech J Anim Sci 9: 277-283.

4. Clark R, Touchberry RW. 1962. Effect of body weight and age at calving on milk production in Holstein cattle. J Dairy Sci 45: 1500-1510. doi: 10.3168/jds.S00220302(62)89662-3

5. Coffey MP, Hickey J, Brotherstone S. 2006. Genetic aspects of growth of Holstein-Friesian dairy cows from birth to maturity. J Dairy Sci 89: 322-329. doi: 10.3168/jds.S0022-0302(06)72097-5

6. Cooke JS, Cheng Z, Bourne NE, Wathes DC. 2013. Association between growth rates, age at first calving and subsequent fertility, milk production and survival in Holstein-Friesian heifers. Open J Anim Sci 3: 1-12. doi: 10.4236/ ojas.2013.31001

7. Evans RD, Wallace M, Garrick DJ, Dillon P, Berry DP, Olori V. 2006. Effects of calving age, breed fraction and month of calving on calving interval and survival across parities in Irish springcalving dairy cows. Livest Sci 100: 216230. doi: 10.1016/j.livprodsci.2005.09.003

8. Ettema JF, Santos JE. 2004. Impact of age at calving on lactation, reproduction, health, and income in firstparity Holsteins on commercial farms. J Dairy Sci 87: 2730-2742. doi: 10.3168/ jds.S0022-0302(04)73400-1

9. Hare E, Norman HD, Wright JR. 2006. Trends in calving ages and calving intervals for dairy cattle breeds in the United States. J Dairy Sci 89: 365-370. doi: 10.3168/jds.S0022-0302(06)72102-6

10. Heinrichs AJ. 1993. Raising dairy replacements to meet the needs of the 21st century. J Dairy Sci 76: 3179-3187. doi: 10.3168/jds.S0022-0302(93)77656-0
11. Heinrichs A, Jones C. 2017. Trends in age at calving of heifers in Pennsylvania. PennState Extension. Pennsylvania, EE.UU. 3 p. [Internet]. Available in: https://extension.psu.edu/trends-in-ageat-calving-of-heifers-in-pennsylvania

12. Hietanen H, Ojala M. 1995. Factors affecting body weight and its association with milk production traits in Finnish Ayrshire and Friesian cows. Acta Agr Scand A-An 45: 17-25. doi: 10.1080/ 09064709509410909

13. [ICBA] Israel Cattle Breeders Association. 2012. The dairy industry in Israel. Israel. [Internet]. Available in: http://www.icba-israel.com/cbase/ 2012.pdf

14. [KNRS] Koninklijk Nederlands Rundvee Syndicaat. 2005. Annual Statistics 2004. Coöperatie Rundveeverbetering Delta, Arnhem, The Netherlands.

15. Lin CY, McAllister AJ, Batra TR, Lee AJ, Roy GL, Vesely JA, Wauthy JM, et al. 1988. Effects of early and late breeding of heifers on multiple lactation performance of dairy cows. J Dairy Sci 71: 2735-2743. doi: 10.3168/jds.S00220302(88)79867-7

16. Meyer M, Everett R, Van Amburgh M. 2004. Reduced age at first calving: effects on lifetime production, longevity and profitability, Arizona Dairy, EE.UU. [Internet]. Available in: http://www.dairyweb.ca/Resources/USWebDocs/ FirstCalvingAge.pdf

17. Mohd Nor N, Steeneveld W, van Werven T, Mourits M, Hogeveen $\mathrm{H}$. 2013. First-calving age and first lactation milk production on Dutch dairy farms. J Dairy Sci 96: 981-992. doi: 10.3168/ jds.2012-5741

18. Moore RK, Kennedy BW, Schaeffer LR, Moxley JE. 1991. Relationships between age and body weight at calving and production in first lactation Ayrshires and Holsteins. J Dairy Sci 74: 269-278. doi: 10.3168/jds.S0022-0302(91)78170-8 
19. Muir B, Fatehi J, Schaeffer L. 2004. Genetic relationships between persistency and reproductive performance in first-lactation Canadian Holsteins. J Dairy Sci 87: 3029-3037. doi: 10.3168/ jds.S0022-0302(04)73435-9

20. Murray B. 2003. Balancing act Research shows we are sacrificing fertility for production traits. Publ. 81-093. Ontario Ministry Agric. Food, Toronto, Canada. [Internet]. Available in: http://www.omafra.gov.on.ca/english/livestock/

21. Pietersma D, Lacroix R, Lefebvre D, Cue R, Wade K. 2006. Trends in growth and age first calving for Holstein and Ayrshire heifers in Quebec. Can J Anim Sci 86: 325-336. doi: 10.4141/A05-080

22. Pirlo G, Miglior F, Speroni M. 2000. Effect of age at first calving on production traits and on difference between milk yield returns and rearing costs on Italian Hols-teins. J Dairy Sci 83: 603-608. doi: 10.3168/jds.S0022-0302(00)74919-8

23. Rokouei M, Torshimi R, Shahrbabak M, Sargolzaei M, Sorencen AC. 2010. Monitoring inbreeding trends and inbreeding depression for economically important traits of Holstein cattle in Iran. J Dairy Sci 93: 3294-3302. doi: 10.3168/ jds.2009-2748

24. Schneider MP, Strandberg E, Emanuelson U, Grandinson K, Roth A. 2007. The effect of veterinarytreated clinical mastitis and pregnancy status on culling in Swedish dairy cows. Prev Vet Med 80: 179-192. doi: 10.1016/ j.prevetmed.2007.02.006

25. Sejrsen K, Purup S, Vestergaard M, Foldager J. 2000. High body weight gain and reduced bovine mammary growth: Physiological basis and implications for milk yield potential. Domest Anim Endocrin 19: 93-104. doi: 10.1016/S0739-7240(00)00070-9
26. Sessarego EA, Vásquez AG, Tello VI. 2014. Evaluación del rendimiento reproductivo del ganado bovino lechero en el valle de Huaura. Spermova 4: 46-49.

27. Simerl NA, Wilcox CJ, Thatcher WW. 1992. Postpartum performance of dairy heifers freshening at young ages. J Dairy Sci 75: 590-595. doi: 10.3168/jds.S00220302(92)77796-0

28. Thompson JR, Pollak EJ, Pelissier CL. 1983. Interrelationships of parturition problems, production of subsequent lactation, reproduction, and age at first calving. J Dairy Sci 66: 1119-1127. doi: 10.3168/jds.S0022-0302(83)-81909-2

29. Tozer PR, Heinrichs AJ. 2001. What affects the costs of raising replacement dairy heifers: A multiple- component analysis. J Dairy Sci 84: 1836-1844. doi: 10.3168/jds.S0022-0302(01)74623-1

30. Wall E, Brotherstone S, Woolliams JA, Banos G, Coffey MP. 2003. Genetic evaluation of fertility using direct and correlated traits. J Dairy Sci 86: 40934102. doi: $10.3168 /$ jds.S0022-0302(03)74023-5

31. Wathes DC, Brickell JS, Bourne NE, Swali A, Cheng Z. 2008. Factors influencing heifer survival and fertility on commercial dairy farms. Animal 2: 11351143. doi: $10.1017 / \mathrm{S} 1751731108002322$

32. Wu JJ, Wathes DC, Brickell JS, Yang LG, Cheng Z, Zhao HQ, Xu YJ, Zhang $S .2012$. Reproductive performance and survival of Chinese Holstein dairy cows. Anim Prod Sci 52: 11-19. doi: 10.1071/ AN11146

33. Zanton GJ, Heinrichs AJ. 2005. Metaanalysis to assess effect of prepubertal average daily gain of Holstein Heifers on first-lactation production. J Dairy Sci 88: 3860-3867. doi: 10.3168/jds.S00220302(05)73071-X 